\title{
Fast and Reusable Facial Rigging and Animation
}

\author{
Verónica Costa Orvalho*, Antonio Susin ${ }^{\dagger}$ \\ Universitat Politècnica de Catalunya* ${ }^{* \dagger}$, Face In Motion*
}

\section{Introduction}

Reproducing the subtleties of a face through animation requires developing a sophisticated character rig. But, creating by hand the inner structure and controls of each character is a very labor-intensive and time-consuming task. We developed an application that is 9099\% faster than traditional manual rigging. The application automatically transfers the rig and animations from the source to a target model. Unlike prior work related to morphing and re-targeting [1] that focus on transferring animations, we transfer the complete facial setup in addition to animations. Our method is general, so artists can define their own rig and then quickly apply it to different models, even with disparate proportions and appearance (human, cartoon or fantastic). This gives artists complete freedom to manipulate the characters: they can create new animations and not be limited by pre-generated ones.

Today, any minor artistic modification that causes the model to change in geometry (a smaller nose, a larger eyebrow) or topology (more resolution around the eyes) leads to the re-starting of most of the rigging process from scratch, making it impossible to re-use the same rig in different face models. We allow re-adjusting the source rig and rapidly propagate the modifications to all the characters. Several videogame and film companies integrated our application into their pipelines and used it for testing: Electronic Arts, Radical Entertainment (Canada) and Dygrafilms (Spain). The tests showed that artists can complete in one hour, tasks that before took them one or two weeks of work; something like changing the weights, modifying an animation control position or transferring animations between characters can be achieved "instantly".

\section{Transferring Facial Rigs and Animations}

Creating and placing by hand each component of the rig (bones, muscles,...) quickly becomes impractical when complexity grows. We created an application in Maya that can handle simple and complex rigs based on a new approach. We begin with two 3D face models. The first one, we call source model, is rigged and includes a series of attributes: a control skeleton, a number of influence objects that represent the inner structure of the face and animation controls, facial expressions (shapes) and animation scripts. The rig doesn't have initial constrains and can be created by an artist. The second model, we call target model, doesn't have a character rig associated to it (figure 1 shows an overview of the process). The source and target models can have different descriptors: one can be defined as a polygonal mesh and the other as a NURBS surface. Also, the faces do not need to have a point-to-point correspondence.

The source rig information is used as the direct input for transferring the setup to the target model. First, the method deforms the source model surface to match the geometry of the target. We landmark the facial features to keep correspondence between source an target model and employ a special case of Radial Basis Function Warping (RBF) as our deformation kernel function. Next, using as reference the previously deformed source surface, we name guide model, the method accurately places the source rig attributes into the target model, even if they have different geometric proportions. We adapted the RBF to properly deal with each attribute specific characteristics. The deformation process achieves excellent results in positioning the source rig attributes in the correct regions of the target face.

After the deformation step comes the skinning, based on a smooth binding algorithm. It binds the transferred attributes to the target model using the adjusted weights of the source, avoiding the need for manual weighting. Last, the method scales and adapts the ani-

*veronica.orvalho@FaceInMotion.com; http://www.FaceInMotion.com mation curves to fit the proportions of the target models. The end result are models ready to be animated with production quality rigs.

\section{Results and Application}

The challenge is to transfer animations from the source and still keep the target model's intensity and look. Our tests compared the target rigs automatically created by the application to the ones manually created by the artists. They showed that our approach is more precise than the artist when adapting the rig to the target geometry, and provides homogeneity in style, which manually is hard to guarantee. If the source rig quality is low, the transference is still successful but results on the target will be of comparable quality.

The main contribution of this application is time saving. Another key feature is the flexibility it allows when defining the facial rig. The modular structure makes it simple to integrate into existing animation pipelines and speed up the overall production. It is now possible to animate characters that before were devoid of expression, because of the time it takes to manually create a rig. Suddenly, all those characters can come alive with the help of our technology.

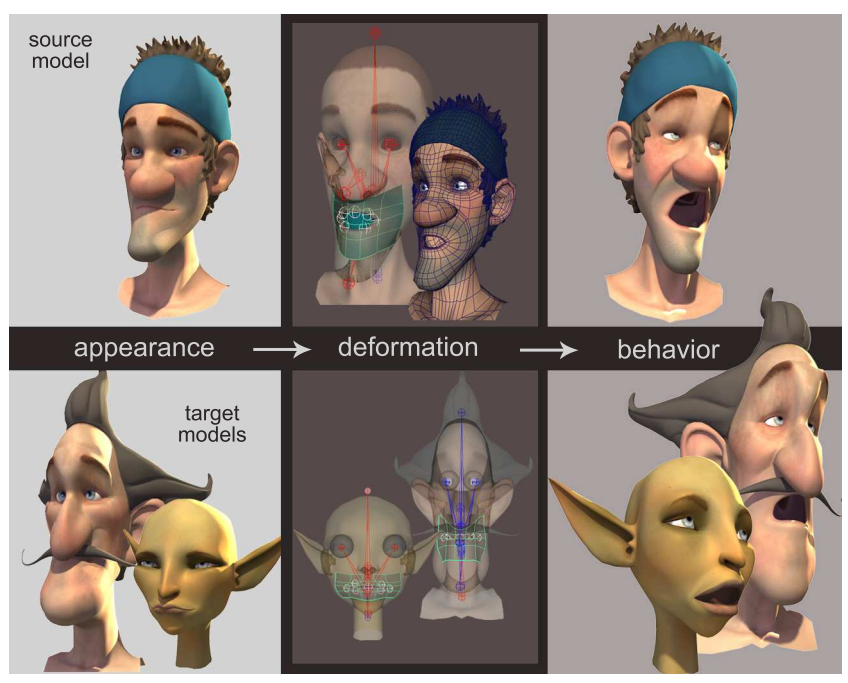

Figure 1: Overview: define the source and target model; adapt the source geometry to fit the target; transfer attributes and shapes; bind the influence objects and skeleton to the target. The result is a model ready to be animated. (Copyright 2005 Dygrafilms)

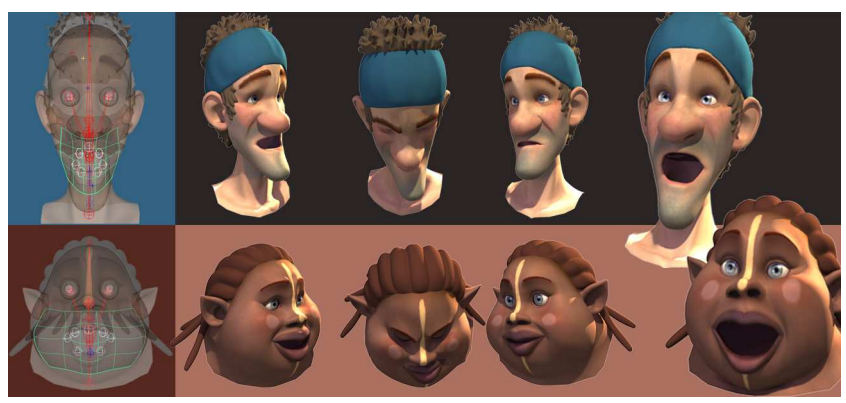

Figure 2: Transfer rig and animations between models: source (first row) and target (second row) have different triangulations. Notice the different proportions of the models: the source has a big nose and skinny face, while the target has a small nose and a fat face. (Copyright 2005 Dygrafilms)

[1] Noh, J.I, Neumann, U. 2001. Expression Cloning. In SIGGRAPH'01 (2001), ACM PREss, pp. 277-288. 